\title{
Self-assembly of colloidal cubes via vertical deposition
}

\author{
Janne-Mieke Meijer ${ }^{*}$, Fabian Hagemans ${ }^{\dagger}$, Laura Rossi ${ }^{\dagger}$, Dmytro V. Byelov ${ }^{\dagger}$, Sonja \\ I.R. Castillo ${ }^{\dagger}$, Anatoly Snigirev ${ }^{\ddagger}$, Irina Snigireva ${ }^{\ddagger}$, Albert P. Philipse ${ }^{\dagger}$, Andrei V. \\ Petukhov ${ }^{\dagger}$
}

$\dagger$ Van 't Hoff Laboratory for Physical and Colloid Chemistry, Debye Institute for Nanomaterials Science, Utrecht University, The Netherlands

†uropean Synchrotron Radiation Facility, Grenoble, France

*Corresponding author. E-mail: J.Meijer1@uu.nl

\section{Supporting Information: The effect of the form factor in the diffraction patterns.}

In figure S1A and S1B the radial averaged scattering intensity profiles of figure $6 \mathrm{~A}$ and $6 \mathrm{~B}$ are displayed on linear and log scale. It can be seen that at low q-values the maximum of the inner Bragg peaks in the top overlaps with a minimum in intensity of the middle region. The opposite occurs for the second Bragg peaks. Both first minima in intensity were determined at $q_{\min }=14,92 \mu \mathrm{m}^{-1}$ for the infiltrated region and at $q_{\min }$

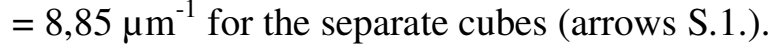

The total diffracted intensity, $I(q)$, is proportional to the product of both the structure factor, $S(q)$, the diffraction of the periodic lattice of a crystal and the form factor, $P(q)$ the intra-particle scattering:

$$
I(q) \propto s(q) P(q)
$$

Here, $P(q)$ will be different for both regions, for the top region the particles are hollow cubes, while in the middle region these are cubic silica shells. The cubes have a superball shape and lie with one flat side on the substrate, which is perpendicular to the beam. Therefore, we approximate the form factor of a rounded cube with that of a sphere; ${ }^{36,37}$,

$$
P_{\text {sphere }}(q, a)=\left[3 \frac{\sin (q a)-q a \cos (q a))^{2}}{(q a)^{5}}\right]^{2}
$$

where the sphere radius, $a$ is approximated as $(D+L) / 4$ and $q$ is the wave vector. For the silica shell, $P(q)$ is approximated as;

$$
P_{\text {shell }}=\frac{1}{V_{\text {shell }}{ }^{2}}\left[V_{\text {total }} P_{\text {sphere }}(q, 0)-V_{\text {iwar }} P_{\text {sphere }}(q, 0)\right]^{2} \text {, }
$$

where $d$ is the outer radius of the shell, $V_{\text {total }}$ is the volume of a the outer sphere with radius $d, V_{\text {inner }}$ is the volume of the inner sphere with radius $a$, and $V_{\text {shell }}=V_{\text {outer }}-V_{\text {inner }}$, the volume of the shell. 
Despite the simplicity of the form factors, they are able to clarify the difference in features observed in the scattering data (Fig. S1B). For instance, the form factor minima can be clearly identified in the averaged radial intensity profiles. The different $P(q)$ furthermore explain the intensity difference of the Bragg spots in both patterns, which are located at corresponding $q$-values. From this we conclude that both regions have the same crystal structure, with $S(q)_{\text {top }}=S(q)_{\text {middle }}$ emphasized differently by the $P(q)$.

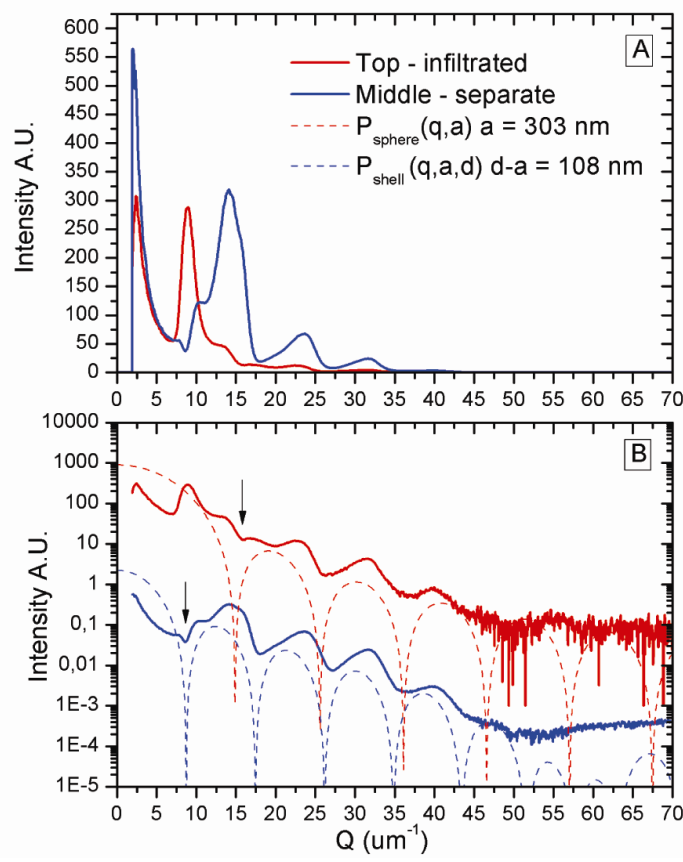

Figure S1. Radial average of 2D SAXS patterns of the infiltrated top region (red) and the noninfiltrated middle (blue) region of the $\Delta T$-sample on A) linear scale and B) on log scale fit. In B) the blue curve is off-set in intensity by factor 1000 for clarity. The curves are fitted with a form factor of a homogeneous sphere and a spherical shell, respectively. 